\title{
Zum Einfluss des \\ Migrationshintergrundes \\ auf das Schüler-Mobbing
}

\section{Lars Oertel, Marlis Schmidt und Wolfgang Melzer}

Die Studie stellt die Situation zu Mobbing-Erfahrungen in den Bundesländern Hessen und Sachsen im Überblick dar und greift dabei auf die Daten der Erhebungswelle 2006 der HBSC-Studie zurück. Es wird untersucht, inwieweit über den Migrationshintergrund der Schüler hinaus andere Variablen der individuellen bzw. der Klassen- und Schulebene Einfluss haben.

Etwa ein Drittel der Befragten gibt an, bereits Mobbing-Erfahrungen gemacht zu haben; ungefähr ein Viertel ist bereits als Mobbing-Täter auffällig geworden. Zudem erweist sich bei den Tätern das Geschlecht, das Alter sowie die Heterogenität bzw. der Migrantenanteil in der Klasse in unterschiedlicher Weise als relevant. Der Migrationshintergrund hat lediglich in Hessen Gewicht. Schüler mit niedrigerem sozioökonomischen Status zeigen ein erhöhtes Risiko, Mobbing-Opfer zu werden. Heterogene Klassen haben in Hessen einen positiven, in Sachsen einen ambivalenten Einfluss.

Dem Migrationshintergrund wird in Schulstudien vergangener Jahre ein hoher Erklärungswert bei Fragen der Bildungsbeteiligung von Kindern und Jugendlichen (Besuch des Gymnasiums) oder des Kompetenzerwerbs im Fachleistungsbereich zugeschrieben (vgl. u.a. Bos et al, 2003; PISA-Konsortium Deutschland, 2007). Auch in der Forschung über Gewalt an Schulen spielt der Migrationshintergrund eine gewisse Rolle, wenn auch nicht in dem Masse, wie man es bei einer Gruppe dieser Grössenordnung, die ca. ein Fünftel der Schülerschaft ausmacht (Walter \& Taskinen, 2007), erwarten könnte.

Ein Problem, das bei Untersuchungen von Schülern mit Migrationshintergrund immer wieder auftaucht und daher umfangreiche Erörterung findet, ist die Abgrenzung dieser Gruppe. Diesbezüglich bemängeln Strohmeier und Spiel (2005) nicht zu Unrecht, dass Definitionen der besagten Gruppe nicht eindeutig seien bzw. gar nicht vorgenommen würden, so dass eine nur begrenzte Vergleichbarkeit der Studien bestehe. Hinzu kommt, dass wegen der Unterschiedlichkeit der Gruppengrössen (nationale Gruppe vs. Gruppe mit Migrationshintergrund) eine Vergleichbarkeit aus methodischen Gründen ohnehin erschwert ist. Ein eben- 
falls zu Recht angemerkter Aspekt ist, dass der Migrationshintergrund oftmals stark mit anderen sozialstrukturellen Merkmalen, wie beispielsweise einer niedrigen Sozialschichtzugehörigkeit korreliert, so dass vermeintlich durch Migration induzierte Abweichungen (sei es bezüglich der Gewalt, aber auch der Schulleistungen) hernach wenig belastbar erscheinen (Fuchs, 1997; Hradil, 2005).

Dennoch sind den vorliegenden Arbeiten zu Migration und Schülergewalt aufschlussreiche Gesichtspunkte zu entnehmen: So registrierte Popp (2000), dass zwischen türkischstämmigen und deutschen Jugendlichen keine signifikanten Unterschiede bei psychischer Gewalt, wohl aber bei zahlreichen anderen Gewaltarten bzw. delinquenten Handlungen zu finden sind. Dabei wurde jeweils immer eine höhere Beteiligung der in der Analyse betrachteten Migrantengruppe registriert. Strohmeier, Atria und Spiel (2005) kommen bei einem Vergleich verschiedener Muttersprachgruppen zu dem Ergebnis, dass Mobbing eher von deutschsprachigen Jugendlichen betrieben wird und bei physischen Attakken vor allem eine Divergenz zwischen den Geschlechtern besteht. In der Untersuchung von Fuchs (1997) ergibt sich dagegen eine signifikant höhere Gewalthäufigkeit von Jugendlichen mit Migrationshintergrund - auch bezüglich psychischer Gewalt, allerdings auf einem geringen Niveau. Es wird gleichwohl darauf hingewiesen, dass dies vornehmlich auf Effekte zurückzuführen ist, die mit den Spezifika dieser Gruppe, also sozialstatistische Unterschiede wie häufigerer Besuch von Hauptschulen etc., korrespondieren, was sich durch ein Abschwächen der Erklärung der Gewalt bei Hinzunahme jener Faktoren abzeichnet. Ähnliche Befunde finden sich bei Schlack und Hölling (2007), die in der Kinder- und Jugendgesundheitsstudie (KIGGS-Studie) des Robert-Koch-Instituts nachwiesen, dass Jugendliche mit Migrationshintergrund häufiger als Täter von Gewalterlebnissen in Erscheinung treten und «permissivere Einstellungen zur Gewalt als [...] Nicht-Migranten haben» (S. 821).

Im Beitrag von Melzer, Bilz und Dümmler (2008) konnte durch die Berücksichtigung des Migrationshintergrundes in der Gruppe der Mobbing-Täter keine zusätzliche Varianz aufgeklärt werden. Ein anderes Bild zeigt sich für die Mobbing-Opfer, da dort die Ergebnisse den Migrationshintergrund als mögliches Risiko für Mobbing-Erfahrungen bei den männlichen Mobbing-Opfern unterstützen. Auch in der Studie von Stein, Dukes und Warren (2007) ordneten sich Kinder mit gemischten Ethnizitäten häufiger der Mobbing-Opfergruppe zu. Dies könnte ein Hinweis darauf sein, dass Kinder mit Migrationshintergrund häufiger Mobbing-Opfer werden. Die Studie von Erhart, Schenk und Ravens-Sieberer (2008) konnte hingegen keine Unterschiede in der Häufigkeit des Mobbings bezogen auf den Migrationshintergrund nachweisen, was sich mit den Ergebnissen anderer internationaler Studien deckt (Seals \& Young, 2003; Stein et al., 2007).

Insgesamt konstatieren bereits Strohmeier, Nestler und Spiel (2005), dass die Heterogenität der Ergebnisse insgesamt darauf hinweist, dass «die kulturellen $\mathrm{Zu}-$ 
gehörigkeiten der Kinder bei aggressiven Konflikten in der Sekundarstufe eine eher untergeordnete Rolle spielen.» (Strohmeier, Nestler \& Spiel 2005, S. 25).

Die Autoren der genannten Untersuchungen gehen fast durchweg von der Annahme aus, der Migrationshintergrund stelle insbesondere im Schulkontext ein erhebliches Risiko dar, Mobbing-Opfer oder -Täter zu werden. Zusätzlich fände sogar noch eine Erhöhung statt, wenn der Anteil der Migranten in der Schulkasse überdurchschnittlich ist und viele verschiedene Migrantengruppen aufeinander treffen (Fuchs, 1997). Damit einhergehend würden sich auch die anderen negativen sozialstrukturellen Merkmale kumulieren, so dass die Schlussfolgerung nahe gelegt wird, dass in Schulklassen mit einem höheren Anteil an Zuwanderern eine höhere Gewaltbereitschaft vorliegt. Diese Konsequenz scheint auch das Kriminologische Forschungsinstitut Niedersachsen [KFN] zu ziehen, wenn es Kinder und Jugendliche mit Migrationshintergrund in ihrem Bericht als eine der vorrangigen Zielgruppen in der Gewaltprävention bezeichnet (2005).

Ein Teil der Widersprüchlichkeit der Befunde kann sicherlich mit dem Hinweis darauf aufgeklärt werden, dass in einigen Untersuchungen delinquente, d.h. härtere und strafbare, Gewalttaten untersucht wurden, während in den Skalen anderer Studien deviante und psychische Gewalt dominierte oder - wie bei unserer Befragung - der Mobbing-Begriff leitend ist.

Interessant sind darüber hinaus Überlegungen der Vertreter der Kontakthypothese, dass gerade die Beziehung und Kommunikation zwischen Personen unterschiedlicher ethnischer Gruppen dazu beiträgt, die Intergruppenbeziehungen zu verbessern und somit einen Abbau diskriminierenden Verhaltens bewirkt (Allport, 1971; Tropp \& Prenovost, 2008). In Schulen, die in ihrer Klassenstruktur ein hohes Mass an kultureller Vielfalt aufweisen, sollten nach dieser Annahme weniger Probleme mit Mobbing aufgrund kultureller und ethnischer Unterschiede auftreten. Tatsächlich weisen Phinney, Ferguson und Tate (1997) nach, dass ethnisch-heterogene ausserschulische Umgebungen über vermehrte Intergruppenkontakte in der Schule zu positiveren Einstellungen gegenüber der anderen Gruppe führen. Auch nach Fritzsche und Wiezorek (2006) neigen (deutsche) Jugendliche, die Freunde anderer kultureller Herkunft haben, weniger zu fremdenfeindlichen Ressentiments. In diesen Fällen spielt jedoch die Freiwilligkeit der Kontakte eine erhebliche Rolle (Jonas, 1998). Im schulischen Kontext, in dem die Klasse eher als Gemeinschaft auf unfreiwilliger Basis betrachtet werden kann, ist dies kaum gegeben. Es ist jedoch nicht auszuschliessen, dass aus dieser alltäglichen Begegnung mit verschiedenartigen kulturellen Einflüssen eine Sensibilisierung für diese möglich erscheint.

Ziel dieser Arbeit ist die Analyse der Einflussfaktoren auf das Mobbing-Geschehen unter besonderer Berücksichtigung des Migrationsstatus'. Vor dem Hinter- 
grund der Kontakthypothese soll zudem eruiert werden, ob der Migrantenanteil einer Schulklasse erklärungsrelevant ist, oder ob es eher andere Faktoren sind, die das Auftreten von Mobbing-Erfahrungen erhöhen. Eine zu diskutierende Fragestellung ist daher, ob eine kulturelle Vielfalt in der Zusammensetzung der Schulklasse einen ausgleichenden bzw. das Mobbing zumindest nicht fördernden Faktor darstellt oder als Konfliktfaktor zu betrachten ist. Dazu werden Befunde zur Mobbing-Erfahrung der Kinder und Jugendlichen mit Migrationshintergrund deskriptiv berichtet, um im späteren Verlauf Zusammenhänge zwischen Mobbing-Erleben und Aspekten der multiethnischen Zusammensetzung von Schulklassen sowie anderer sozialstatistischer Faktoren mittels logistischer Regressionsanalysen zu überprüfen.

\section{Methode}

\section{St ichprobe}

Basis der Untersuchung ist der Datensatz aus dem Jahr 2006 der WHO-Studie «Health Behaviour in School-aged Children» (HBSC). Dieser international angelegte Survey wird im Abstand von vier Jahren in fast allen europäischen Ländern, den USA, Kanada und Israel durchgeführt und erhebt mittels eines standardisierten Fragebogens das Gesundheitsverhalten und die subjektiv berichtete Gesundheit von Kindern und Jugendlichen in den fünften, siebten und neunten Klassen. Im Rahmen dessen wurden für die deutschen Bundesländer Berlin, Hamburg, Nordrhein-Westfahlen, Hessen und Sachsen auch Daten bezüglich des Erlebens von Mobbing als Opfer bzw. als Täter erfragt.

Für unsere Fragestellung war es vornehmlich interessant, ob eine unterschiedliche Zusammensetzung der Schülerschaft (bezogen auf den Migrationshintergrund) auch ein unterschiedliches Mobbingerleben induziert. Hierbei bieten sich die Datensätze aus Sachsen und Hessen an, da sich diese einerseits als Flächenländer relativ ähneln und andererseits die Daten eines ostdeutschen Bundeslandes eine weit geringere Anzahl von Schülern mit Migrationshintergrund in den Klassen erwarten lässt, wohingegen eine typische Schulklasse in Hessen eine für den Westen Deutschlands charakteristische Zusammensetzung widerspiegeln sollte. Für eine realitätsnahe Abbildung werden entsprechend der jeweiligen Schulstruktur zufällig Schulen ausgewählt, von welchen dann alle Schüler aus den Klassen der gesuchten Jahrgänge befragt werden. Insgesamt gehen 4367 Schülerinnen und Schüler für Sachsen und 3020 für Hessen in die Berechnungen ein.

Durch die Grösse der Befragung dürfte zudem die Möglichkeit bestehen, dass auch in Sachsen für eine tiefer gehende statistische Auswertung ausreichend Kinder und Jugendliche mit Migrationshintergrund vertreten sind. 
Die Stichprobe setzte sich aus Schülern der fünften, siebten und neunten Klasse der Bundesländer Hessen (59.1\%) und Sachsen (40.9\%) zusammen, wobei der Altersdurchschnitt bei $\mathrm{M}=14.0$ (SD=1.87, $\mathrm{Min}=10, \mathrm{Max}=19)$ liegt. 50.2\% der Schüler sind Jungen und 49.8\% Mädchen.

Tabelle 1 zeigt die Stichprobenzusammensetzung bezüglich des Migrationshintergrundes. Dabei wird der bereits eingangs vermutete Unterschied bezüglich des Anteils der Kinder mit Migrationshintergrund in den Bundesländern bestätigt. Ersichtlich wird, dass in Hessen sowohl in der Schülergeneration jedoch besonders in der Elterngeneration deutlich mehr Personen einen Migrationshintergrund aufweisen. Die Unterschiede zwischen den Ländern sind diesbezüglich auf deskriptiver Ebene signifikant. Ähnliches zeigt sich für die zu Hause gesprochene Sprache, die in Sachsen häufiger Deutsch ist. Berücksichtigt man alle Faktoren, die einen Migrationshintergrund in der 1. oder 2. Generation anzeigen, kann zusammenfassend gesagt werden, dass 33.6\% der Schüler in Hessen und $11.8 \%$ der Schüler in Sachsen einen Migrationshintergrund haben, wobei der Anteil in Hessen signifikant höher ist.

Tabelle 1: Analyseebenen der logistischen Regression

\begin{tabular}{|c|c|c|c|c|}
\hline Variable & Modell 1 & Modell 2 & Modell 3a & Modell 4 \\
\hline Migrationshintergrund Nicht-Migrant (Referenz) & \multirow[t]{8}{*}{$\mathrm{X}$} & \multirow[t]{2}{*}{$\mathrm{X}$} & \multirow[t]{2}{*}{$\mathrm{X}$} & \multirow[t]{2}{*}{$\mathrm{X}$} \\
\hline Individuelle Ebene & & & & \\
\hline Geschlecht weiblich(Referenz) & & $\mathrm{X}$ & $\mathrm{X}$ & $\mathrm{X}$ \\
\hline Alter 10 Jahre (Referenz) & & $\mathrm{X}$ & $\mathrm{X}$ & $\mathrm{X}$ \\
\hline Sprache Deutsch (Referenz) & & $\mathrm{X}$ & $\mathrm{X}$ & $\mathrm{X}$ \\
\hline FAS hoch (Referenz) & & \multirow[t]{3}{*}{$\mathrm{X}$} & \multirow[t]{2}{*}{$\mathrm{X}$} & \multirow[t]{2}{*}{$\mathrm{X}$} \\
\hline Klassenebene & & & & \\
\hline $\begin{array}{r}\text { Migrantenanteil in der Klasse keine Migranten } \\
\text { (Referenz) }\end{array}$ & & & $\mathrm{X}$ & $\mathrm{X}$ \\
\hline Schulebene (nur in Sachsen) & & & & \\
\hline Schulform Gymnasium (Referenz) & & & & $X$ \\
\hline
\end{tabular}

\section{Messinstrumente}

Die der HBSC-Studie zu Grunde liegende Definition von Mobbing geht auf Dan Olweus (2002) zurück und wurde dem Begriffsverständnis der 11 bis 16-jährigen Schülerinnen und Schüler in «Schikanieren» übersetzt. Von Mobbing wird gesprochen, wenn ein Schüler oder eine Schülerin wiederholt und über einen längeren Zeitraum negativen Handlungen, also Verletzungen und Unannehmlichkeiten wie Drohen, Spotten, Hänseln, Beschimpfen, aber auch sozialen Ausgrenzungen, ausgesetzt ist. Dabei ist zu berücksichtigen, dass ein Ungleichgewicht zwischen Täter(n) und Opfer(n) besteht (Olweus, 2002). 
Die Mobbing-Erfahrungen wurden mittels zweier Items erfasst a) Wie oft bist $\mathrm{du}$ in den letzten Monaten von Mitschülern schikaniert worden? und b) Wie oft hast du in den letzten Monaten dabei mitgemacht, wenn jemand in der Schule schikaniert wurde? Die Antwort erfolgte auf einer 5-stufigen Skala, die nachträglich dichotomisiert wurde, d.h. die beiden ersten und die drei letzten Kategorien des 5-stufigen Antwortformates («nie», «ein- bis zweimal», «zwei- bis dreimal im Monat» «ungefähr einmal pro Woche» und «mehrmals pro Woche») bildeten zwei neue Kategorien, so dass eine eindeutige Zuteilung jedes Schülers hinsichtlich der Kategorien a) Mobbing-Opfer vs. kein Mobbing-Opfer und b) Mobbing-Täter vs. kein Mobbing-Täter vorgenommen werden konnte. Bei der Einteilung wurde die exponierte Gruppe der Täter-Opfer nicht berücksichtigt. Die Mitglieder dieser Gruppe lassen sich neben einem geringen Selbstkonzept vor allem dadurch charakterisieren, dass sie Formen härterer Gewalt bevorzugen, unaufmerksam sind, zu Hyperaktivität neigen und impulsiv auftreten (Olweus, 2002). Aufgrund dieser Spezifika, die nicht im Zentrum des vorliegenden Mobbing-Begriffs stehen und durch die vorgelegten Items nur partiell erfasst werden, konzentrieren sich die Betrachtungen in dieser Untersuchung auf «reine» Täter bzw. Opfer. Eine Einbeziehung dieser kleinen, jedoch für den Schulalltag aufgrund ihrer Typologie sehr bedeutsamen Gruppe (2.3\%) der Täter-Opfer bedürfte einer gesonderten Betrachtung, die hier nicht gewährleistet werden kann, so dass sie - auch angesichts modell-theoretischer Überlegungen (Melzer, Bilz \& Dümmler, 2008) - aus den folgenden Analysen ausgeklammert bleibt.

Um den genannten Problemen bezüglich der Einteilung von Schülern mit und ohne Migrationshintergrund im Sinne der entwickelten Forschungsfragen zu begegnen, wurden alle Schüler, die entweder selbst nicht in Deutschland geboren wurden oder von denen mindestens ein Elternteil im Ausland geboren wurde oder deren Sprache zu Hause nicht deutsch ist -immerhin sprechen fast die Hälfte aller Schülerinnen und Schüler mit Migrationshintergrund nicht deutsch (Walter \& Taskinen, 2007) -, zur Gruppe der Schüler mit Migrationshintergrund zugeordnet. Dadurch werden alle Schüler identifiziert, die sich durch die kulturelle Praxis, die für die Fragestellung bezüglich der kulturellen Heterogenität bedeutend ist, von den deutschen Schülern unterscheiden (Esser, 2001). Schliesslich zeigt sich am Gebrauch der Sprache, in welcher Stärke die jeweiligen Schüler die «Heimatkultur» in den Kontext der Schulklasse einbringen. Dies ist insbesondere bei der Beantwortung der Fragen wichtig, bei denen es um die Anzahl unterschiedlicher Kulturen geht, die sich in einer Klasse zusammenfinden. Die Berücksichtigung der Sprache für die Feststellung des Migrationshintergrundes schliesst damit an andere Untersuchungen an.

Für die Beantwortung der eingangs erwähnten Forschungsfragen war es zudem relevant, die Zusammensetzung einer Schulklasse, die ein Schüler besucht, hinsichtlich des Migrationshintergrundes zu betrachten. Um die entsprechenden Berechnungen zu ermöglichen, war es notwendig, eine Kategorisierung vorzu- 
nehmen. Dazu wurde der Anteil von Migranten pro Klasse berechnet. Anschliessend wurden aufgrund der deskriptiven Verteilung in der Stichprobe die Kategoriengrenzen so gewählt, dass die unterschiedlichen Migrantenanteile in Sachsen und Hessen berücksichtigt werden konnten und nahezu eine Gleichverteilung für alle Kategorien erreicht wurde.

Weitere Prädiktoren waren das Geschlecht, das Alter, die zu Hause gesprochene Sprache und der sozioökonomische Status, welcher in der HBSC-Studie mit Hilfe des dreistufigen FAS-Index' (=family affluence scale, Massstab des familiären Wohlstands) bestimmt wird (Boyce, Torsheim, Currie \& Zambon, 2006).

\section{Analyseme thode}

Um den Einfluss der zuvor besprochenen möglichen Prädiktoren im Hinblick auf die Erfahrungen als Mobbing-Opfer und Mobbing-Täter zu untersuchen, werden logistische Regressionen gerechnet. Dies ist möglich, weil durch die bereits erwähnte Dichotomisierung der Mobbing-Skala seltene und gelegentliche Mobbingerfahrungen von häufigen und regelmässigen geschieden werden. Für die Interpretation werden die Odds-Ratios herangezogen, die Aussagen darüber zulassen, inwiefern sich die Wahrscheinlichkeit, Mobbing-Opfer bzw. MobbingTäter zu werden, unter Berücksichtigung der Einflussfaktoren verändert. In diesem Sinne können die Odds-Ratios also ähnlich den gängigen Effektstärken interpretiert werden (Nachtigall \& Wirtz, 1998).

Durch die Hinzunahme unterschiedlicher Faktoren in einzelnen Modellen soll sich zeigen, inwiefern sich die Vorhersagkraft von Mobbing-Erfahrungen sukzessive verbessern lässt. Im Modell 1 wird zunächst nur der Migrationshintergrund der Schüler gesondert betrachtet. Im folgenden Modell 2 werden weitere Variablen berücksichtigt, die auf der individuellen Ebene angesiedelt sind. Das Modell 3 enthält einen Faktor, der die Zusammensetzung der Klasse charakterisiert, also den Anteil von Schülern mit Migrationhintergrund in der Klasse beschreibt. Im Modell 4 soll untersucht werden, ob mit Hilfe der Schulform die Vorhersage von Mobbing-Erfahrungen verbessert werden kann. In Tabelle 2 sind die einzelnen Anlayseebenen im Überblick dargestellt.

Aufgrund der Grösse der Stichprobe wird das $\alpha$-Fehler-Niveau auf $0.1 \%$ festgelegt (Bortz, 2005). 
Tabelle 2: Migrationshintergrund der Schüler in Sachsen $(N=4367)$ und Hessen $(N=3020)$

\begin{tabular}{|c|c|c|c|c|c|}
\hline \multirow[t]{2}{*}{ Variable } & & \multicolumn{3}{|c|}{ Region } & \multirow[t]{2}{*}{$\chi^{2}$-Test } \\
\hline & & Hessen & Sachsen & gesamt & \\
\hline Migrationshintergrund & $j a$ & $6.1 \%$ & $4.6 \%$ & $5.2 \%$ & $.005^{* *}$ \\
\hline Kind & nein & $93.9 \%$ & $95.4 \%$ & $94.8 \%$ & $(\mathrm{~F}=7.970 \mathrm{df}=1)$ \\
\hline Migrationshintergrund & $j a$ & $26.3 \%$ & $8.3 \%$ & $15.7 \%$ & $.000^{* * *}$ \\
\hline Mutter & nein & $73.7 \%$ & $91.7 \%$ & $84.3 \%$ & $(\mathrm{~F}=437.782 \mathrm{df}=1)$ \\
\hline Migrationshintergrund & $j a$ & $28.2 \%$ & $10.2 \%$ & $17.6 \%$ & $.000^{* * *}$ \\
\hline Vater & nein & $71.8 \%$ & $89.8 \%$ & $82.4 \%$ & $(\mathrm{~F}=397.780 \mathrm{df}=1)$ \\
\hline \multirow[t]{3}{*}{ Sprache zu Hause } & Deutsch & $87.3 \%$ & $96.1 \%$ & $92.5 \%$ & $.000^{* * *}$ \\
\hline & nicht Deutsch & $5.3 \%$ & $3.9 \%$ & $4.4 \%$ & $(\mathrm{~F}=347.512 \mathrm{df}=2)$ \\
\hline & keine Angabe & $7.4 \%$ & $.0 \%$ & $3.0 \%$ & \\
\hline Migrationshintergrund & $j a$ & $33.8 \%$ & $11.8 \%$ & $20.8 \%$ & $.000^{* * *}$ \\
\hline gesamt & nein & $66.2 \%$ & $88.2 \%$ & $79.2 \%$ & $(\mathrm{~F}=525.018 \mathrm{df}=1)$ \\
\hline
\end{tabular}

${ }^{*} \mathrm{p} \leq .05,{ }^{* *} \mathrm{p} \leq .01^{* * *} \mathrm{p} \leq .001$

\section{Ergebnisse}

\section{Mobbing}

In Tabelle 3 sind die Ergebnisse zum Schüler-Mobbing zusammengefasst. Es wird deutlich, dass mehr als zwei Drittel der Kinder in den letzten Monaten gar nicht gemobbt wurde. Dies bedeutet allerdings auch, dass ca. 30\% Mobbing-Erfahrungen gemacht haben. Der Grossteil dieser Gruppe (20.4\%) wurde in den letzten Monaten einmal gemobbt. Allerdings werden auch 2.9\% der Kinder einmal pro Woche bzw. 3.6\% sogar mehrfach pro Woche von anderen gemobbt. Im Vergleich der Bundesländer wird deutlich, dass in Sachsen mehr Schüler angeben, in den letzten Monaten gar nicht gemobbt worden zu sein.

Weiterhin treten 64.6\% nie als Mobbing-Täter in Erscheinung. Etwa ein Viertel hat in den letzten Monaten zumindest einmal einen anderen Schüler gemobbt; jeweils 2.6\% tun dies einmal in der Woche oder sogar mehrfach pro Woche. Die deskriptiven Angaben in den Bundesländern unterscheiden sich hier nicht signifikant. 
Tabelle 3: Mobbing-Erfahrung der Schüler in Sachsen $(N=4367)$ und Hessen $(N=3020)$

\begin{tabular}{|ll|cccc|}
\hline Variable & & $\begin{array}{c}\text { Region } \\
\text { Hessen }\end{array}$ & Sachsen & gesamt & \multirow{2}{*}{$\chi^{2}$-Test } \\
\hline Mobbing-Opfer & Nicht gemobbt & $65,1 \%$ & $69,7 \%$ & $67,8 \%$ & \\
& 1-2 mal & $21,1 \%$ & $19,2 \%$ & $20,0 \%$ & $.001^{* * *}$ \\
& 2-3 mal pro Monat & $4,9 \%$ & $3,9 \%$ & $4,3 \%$ & $(\mathrm{~F}=19,025 \mathrm{df}=4)$ \\
& ca. 1 mal pro Woche & $4,0 \%$ & $3,3 \%$ & $3,6 \%$ & \\
& mehrmals pro Woche & $4,9 \%$ & $4,0 \%$ & $4,4 \%$ & \\
\hline Mobbing-Täter & habe nicht gemobbt & $62,5 \%$ & $63,5 \%$ & $63,1 \%$ & \\
& 1-2 mal & $25,9 \%$ & $26,4 \%$ & $26,2 \%$ & \\
& 2-3 mal pro Monat & $4,4 \%$ & $4,3 \%$ & $4,3 \%$ & (F=7, $556 \mathrm{df}=4)$ \\
& ca. 1 mal pro Woche & $3,5 \%$ & $3,2 \%$ & $3,3 \%$ & \\
& mehrmals pro Woche & $3,8 \%$ & $2,7 \%$ & $3,1 \%$ & \\
\hline
\end{tabular}

${ }^{*} \mathrm{p} \leq .05,{ }^{* *} \mathrm{p} \leq .01^{* * *} \mathrm{p} \leq .001$

\section{Mobbing-Opfer}

Im Modell 1 zeigt sich, dass der Migrationshintergrund weder in Hessen noch in Sachsen die Wahrscheinlichkeit Mobbing-Opfer zu werden, erhöht (Tabelle 4).

Im zweiten Modell erweist sich lediglich der sozioökonomische Status als statistisch relevant für die Vorhersage der Risiko-Gruppen-Zugehörigkeit. Die Chance eines Jugendlichen Mobbing-Opfer zu werden, erhöht sich in Sachsen fast um das Doppelte $(\mathrm{OR}=1.89)$, wenn dieser einen niedrigen sozioökonomischen Status besitzt. In Hessen stellt besonders ein mittlerer sozioökonomischer Status ein Risiko dar, da hier die Wahrscheinlichkeit, Mobbing-Opfer zu werden signifikant zunimmt. Alle anderen Faktoren, wie das Alter und das Geschlecht, erweisen sich als nicht signifikant. Der Sprachgebrauch der Schüler bleibt ebenfalls ohne Einfluss.

Berücksichtigt man Prädiktoren, die Aussagen über die Zusammensetzung einer Klasse machen können, so zeigen sich für die Bundesländer folgende Effekte: Sowohl in Hessen als auch in Sachsen trägt der Migrantenanteil der Klasse nicht weiter zur Vorhersage der Mobbing-Opfer-Wahrscheinlichkeit bei. Zwar zeigen sich besonders in der hessischen Stichprobe Veränderungen in den Odds-Ratios, die auf ein zunehmendes Risiko für Mobbing-Opfer in Klassen mit hohem Migrantenanteil hinweisen, allerdings sind diese nicht signifikant.

Bei der Betrachtung des die Schulform beinhaltenden Modells in Sachsen, lässt sich diagnostizieren, dass es ein erhöhtes, jedoch nicht signifikantes Risiko für Mittelschüler des Realschulzweiges gibt (OR=1.39), Mobbing-Opfer zu werden. 
Tabelle 4: Logistische Regression zum Zusammenhang von Mobbing und Migrationshintergrund in Sachsen $(N=4169)$

\begin{tabular}{|c|c|c|c|c|c|c|c|c|c|}
\hline \multirow[b]{2}{*}{ Variable } & & \multicolumn{2}{|c|}{ Modell 1} & \multicolumn{2}{|c|}{ Modell 2} & \multicolumn{2}{|c|}{ Modell 3} & \multicolumn{2}{|c|}{ Modell 4} \\
\hline & & Opfer & Täter & Opfer & Täter & Opfer & Täter & Opfer & Täter \\
\hline $\begin{array}{r}\text { Migrations- } \\
\text { hintergrund } \\
\text { Nicht-Migrant } \\
\text { (Referenz) }\end{array}$ & Migrant & 1.05 & .91 & 1.09 & .99 & 1.14 & 1.01 & 1.13 & .1 .00 \\
\hline $\begin{array}{l}\text { Individuelle } \\
\text { Ebene } \\
\qquad \begin{array}{r}\text { Gescblecht } \\
\text { weiblich } \\
\text { (Referenz) }\end{array}\end{array}$ & männlich & & & 1.05 & $2.19^{* * *}$ & 1.05 & $2.19^{* * *}$ & 1.04 & $2.16^{* * *}$ \\
\hline $\begin{array}{r}\text { Alter } \\
10 \mathrm{Jahre} \\
\text { (Referenz) }\end{array}$ & 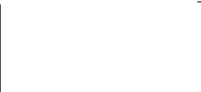 & & &. .97 & $1.27^{* * *}$ & .96 & $1.28^{* * *}$ & .95 & $1.27^{* * *}$ \\
\hline $\begin{array}{r}\text { Sprache } \\
\text { Deutsch } \\
\text { (Referenz) }\end{array}$ & andere Sprache & & & .62 & .90 & .60 & .89 & .63 & .93 \\
\hline $\begin{array}{r}F A S \\
\text { hoch } \\
\text { (Referenz) }\end{array}$ & $\begin{array}{r}\text { mittel } \\
\text { niedrig }\end{array}$ & & & $\begin{array}{c}1.30^{*} \\
1.79^{* * *}\end{array}$ & $\begin{array}{l}.99 \\
.73\end{array}$ & $\begin{array}{c}1.29^{*} \\
1.75^{* * *}\end{array}$ & $\begin{array}{r}100 . \\
.74\end{array}$ & $\begin{array}{l}1.26^{*} \\
1.68^{* *}\end{array}$ & $\begin{array}{l}.98 \\
.72\end{array}$ \\
\hline $\begin{array}{l}\text { Klassenebene } \\
\text { Migrantenanteil } \\
\text { in der Klasse } \\
\text { keine Migranten } \\
\text { (Referenz) }\end{array}$ & $\begin{array}{r}0,1 \% \text { bis unter } 5 \% \\
5 \% \text { bis unter } 10 \% \\
10 \% \text { bis unter } 20 \% \\
20 \% \text { bis unter } 30 \% \\
30 \% \text { bis unter } 50 \% \\
\text { über } 50 \%\end{array}$ & & & & & $\begin{array}{l}1.05 \\
.86 \\
.64^{* *} \\
.83 \\
1.34 \\
.49\end{array}$ & $\begin{array}{c}1.03 \\
1.55^{* *} \\
.967 \\
.944 \\
1.26 \\
.89\end{array}$ & $\begin{array}{c}1.07 \\
.88 \\
.65^{* *} \\
.81 \\
1.27 \\
.44\end{array}$ & $\begin{array}{c}1.04 \\
1.60^{* *} \\
1.00 \\
.94 \\
1.22 \\
.82\end{array}$ \\
\hline $\begin{array}{l}\text { Schulebene } \\
\text { Schulform } \\
\text { Gymnasium } \\
\text { (Referenz) }\end{array}$ & $\begin{array}{r}\text { Realschule } \\
\text { Hauptschule }\end{array}$ & & & & & & & $\begin{array}{l}1.27^{*} \\
1.02\end{array}$ & $\begin{array}{l}1.23 \\
1.07\end{array}$ \\
\hline $\begin{array}{l}\text { Nagelkerkes } \\
\mathrm{R}^{2} \text { / Erklärte } \\
\text { Varianz }\end{array}$ & & .000 & .000 & $.008^{*}$ & $.061^{* * *}$ & $.018^{* * *}$ & $.068^{* * *}$ & $.020^{* * *}$ & $.069^{* * *}$ \\
\hline
\end{tabular}

${ }^{*} \mathrm{p} \leq .05,{ }^{* *} \mathrm{p} \leq .01^{* * *} \mathrm{p} \leq .001$ 


\section{Mobbing-Täter}

Zunächst werden die Ergebnisse der Bundesländer unter Berücksichtigung der unterschiedlichen Faktoren in den drei Modellen gegenübergestellt (Tabelle 5). Der Migrationsstatus spielt auch hier in Sachsen keine Rolle. Allerdings erhöht sich bei Schülern in Hessen unter Berücksichtigung des Migrationsstatus' die Chance Mobbing-Täter zu werden um das 1.54-fache.

Im Modell 2 wird besonders der Einfluss des Geschlechts deutlich. Jungen haben in Sachsen ein 1.93-fach, in Hessen sogar ein 3.56-fach erhöhtes Risiko, als Mobbing-Täter in Erscheinung zu treten. Zudem zeigen sich in beiden Bundesländern mit Odds-Ratios von $\mathrm{OR}=1.32$ (Sachsen) und $\mathrm{OR}=1.31$ (Hessen) ähnlich höhere Wahrscheinlichkeiten für aktives Mobbing mit zunehmendem Alter. Am Beispiel bedeutet dies, dass ein 15-jähriger Jugendlicher in Sachsen im Vergleich zu einem Zehnjährigen eine 3.94-fache Wahrscheinlichkeit hat ein Mobbing-Täter zu werden. In Hessen beträgt der Faktor für dieselbe Altersspanne $\mathrm{OR}=3.9$. Der sozioökonomische Status hat in beiden Bundesländern keinen Einfluss auf das Risiko, Mobbing-Täter zu werden.

Im Modell 3 sind, unter Berücksichtigung des Migrantenanteils der Klasse, in beiden Bundesländern unterschiedliche Effekte zu verzeichnen. In Sachsen weisen Mobbing-Täter in Klassen mit einem recht geringen Anteil von $45,1 \%$ bis 10\%» ein 2-fach erhöhtes Risiko auf, Mobbing-Täter zu werden. In allen anderen Klassen gibt es bezüglich des Migrantenanteils der Klasse keine bedeutsamen Hinweise auf eine Erhöhung des Risikos Mobbing-Täter zu werden. In Hessen gibt es keinen signifikanten Einfluss des Migrantenanteils der Klasse auf die Chancen Mobbing-Täter zu werden, wobei jedoch ein Absinken der Odds-Ratios verzeichnet werden kann.

Schliesslich sei festgestellt, dass die in Modell 4 einbezogene Schulform (in Sachsen) keine nennenswerten Veränderungen im Vergleich zum Modell 3 offenbart. 
Tabelle 5: Logistische Regression zum Zusammenhang von Mobbing und Migrationshintergrund in Hessen $(N=2886)$

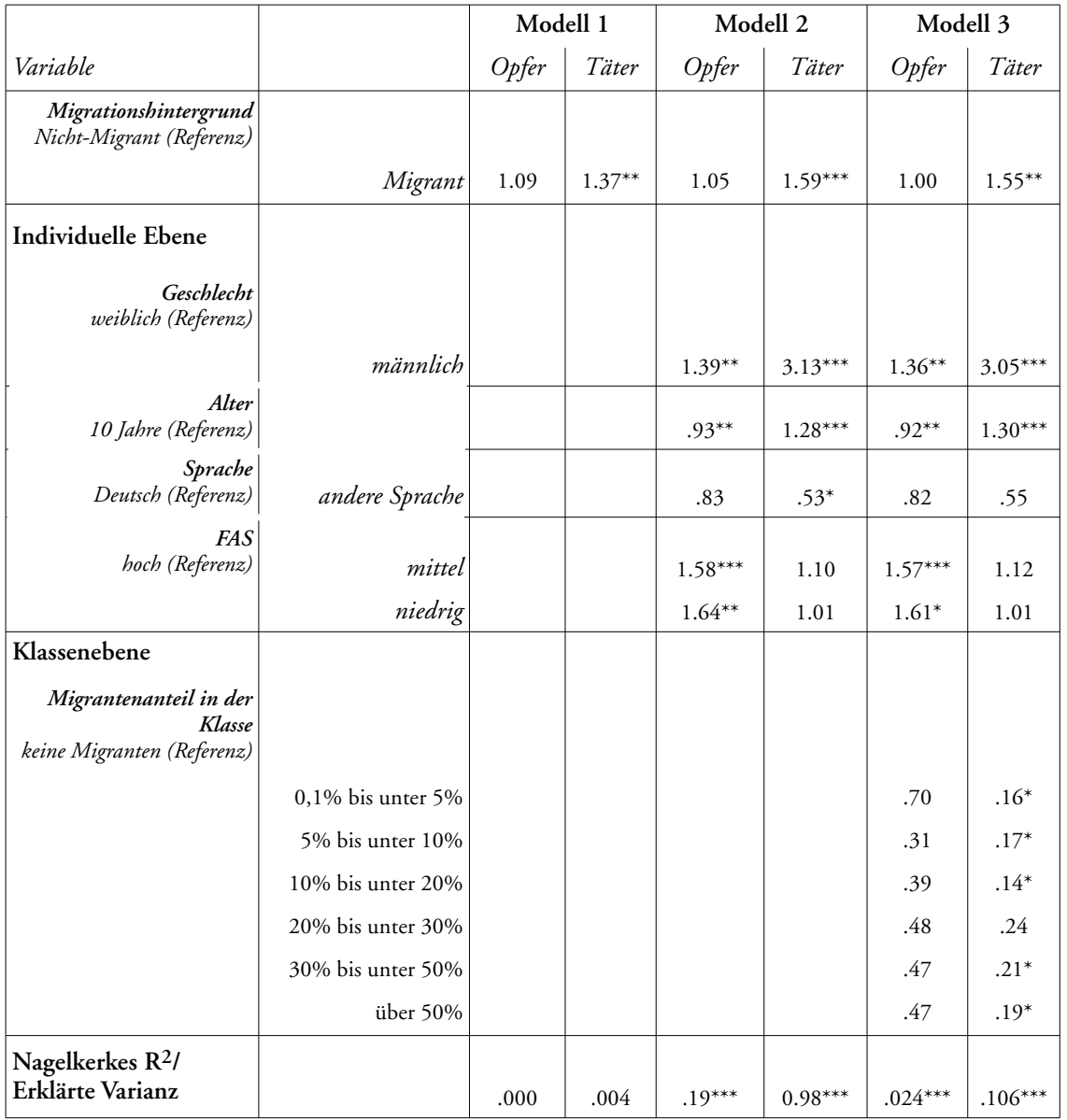

Anmerkungen: die Schulebene entfällt hier, da in Hessen keine Angaben zur Schulform erhoben wurden ${ }^{*} \mathrm{p} \leq .05,{ }^{* *} \mathrm{p} \leq .01^{* * *} \mathrm{p} \leq .001$

\section{Diskussion}

Die Aufgliederung der Prädiktoren in eine individuelle Ebene und die Klassenebene ermöglicht es, detaillierte Aussagen zu treffen, ob das berichtete MobbingVerhalten bzw. -Erleben eher auf Ursachen zurückzuführen ist, die das schulische Umfeld betreffen, mit dem der Schüler oder die Schülerin zwangsläufig konfrontiert wird, oder ob es Gründe sind, die eher in der Person bzw. in herkunftsspezifischen Gesichtspunkten begründet liegen. 
Bei der Betrachtung der Ergebnisse in den beiden Bundesländern ist jeweils immer deren eingangs erwähnte Verschiedenartigkeit hinsichtlich des Migrantenanteils zu beachten. Speziell für Sachsen bedeutet das, dass beim vergleichsweise geringen Migrantenanteil in der Bevölkerung bereits dadurch gänzlich andere Voraussetzungen für das Gros der Schüler besteht, die das Erlernen toleranter Einstellungen beeinflussen können.

Eine zentrale Fragestellung der vorliegenden Studie war es, zu untersuchen, inwieweit sich die multiethnische Zusammensetzung einer Klasse, entgegen den bisherigen Annahmen der Literatur, positiv auf die Mobbing-Erfahrungen im Sinne einer niedrigeren Prävalenz auswirken kann.

Für die Mobbing-Opfer bleibt festzuhalten, dass in beiden Bundesländern der Migrantenanteil keinen signifikanten Einfluss hat. Das bedeutet zwar, dass die These einerseits nicht bestätigt werden kann. Andererseits ist festzuhalten, dass ein höherer Anteil an Migranten in einer Klasse auch nicht zu einer Erhöhung des Mobbing-Opfer-Anteils führt. Als Erklärung im Sinne der Zielformulierung dieser Arbeit wäre es denkbar, dass die Erhöhung des Konfliktpotentials, die die Erhöhung des Migrantenanteils mit sich bringt, durch ein gleichzeitiges (zwangsläufiges) Erlernen toleranter Einstellungen forciert wird und somit das Risiko etwas mindert, Mobbing-Opfer zu werden.

Bemerkenswert ist der Einzelbefund für Sachsen, bei dem die Chance Mobbing-Täter zu werden in Klassen mit einem geringem Migrantenanteil in der Kategorie « $5,1 \%$ bis 10\%» signifikant zunimmt. Dies kann zum einen an der Stichprobenzusammensetzung liegen; zum anderen wäre es denkbar, dass ein geringer Migrantenanteil in der Klasse nicht genügend Interaktionsmöglichkeiten bietet und dadurch die Intention der Kontakthypothese nicht zum Tragen kommen kann, nach der ein Abbau von Vorurteilen und diskriminierendem Verhalten nur dann möglich ist, wenn ausreichend Möglichkeit zu positivem Kontakt besteht. Dieser muss auch in Klassen mit hohem Migrantenanteil nicht gewährt sein. Überwiegen beispielsweise negative Interaktionen den Kontakt, so kann laut Allport (1971) keine Reduzierung der Intergruppenkonflikte und somit eine positive Interaktion erreicht werden. Ein Hinweis hierauf könnten die Trends der Odds-Ratios für Mobbing-Opfer in den Kategorien «30.1\% bis $50 \%$ » und "über 50\%» in Hessen sein.

Weiterhin zeigt sich in Hessen, bezogen auf die Fragestellung hinsichtlich der interkulturellen Vielfalt der Klassen, ein durchweg positiver Trend des Migrantenanteils für die Mobbing-Täter. Die Daten zeigen, dass psychische Gewaltformen in solchen Klassen keinesfalls vermehrt auftreten, was zumindest in Hessen mit einiger Sicherheit berichtet werden kann. Die interkulturelle Vielfalt einer Klasse offenbart in diesem Bundesland in Bezug auf Mobbing-Täterschaften einen durchweg dämpfenden Effekt. Hinzu kommt, dass die Kontakte in Schulen nicht notwendig freiwilliger Natur sind und andere Faktoren des Schulklimas, wie Leistungsdruck oder das Unterstützungsverhalten der Lehrer und Mitschüler, sich auf den Umgang der Schüler miteinander auswirken können. 
Zudem stellt sich die Frage, ob der Migrantenanteil ein geeignetes Mass ist, mit dem die multiethnische Vielfalt einer Klasse beschrieben werden kann. Es ist zu überlegen, ob es einen Unterschied macht, wenn in einer Klasse mit 30\% Migrantenanteil, diese alle denselben Migrationhintergrund (bspw. Türkei) aufweisen, oder ob sich der Migrantenanteil aus Kindern mit unterschiedlichen Herkunftsländern zusammensetzt. Letzteres wäre vermutlich durch ein höheres Mass an kultureller Vielfalt gekennzeichnet. Den Einfluss eines solchen Koeffizienten über den Migrantenanteil der Klasse hinaus in speziellen Studien zu Mobbing zu untersuchen, scheint somit notwendig, da die vorliegende Studie mit ihrem Fokus auf das Gesundheitsverhalten von Kindern und Jugendlichen dies nicht zu leisten vermag.

Ein interessantes Ergebnis bezüglich des Migrationshintergrundes der Schüler zeigte sich bei den Mobbing-Tätern, da dieser in Hessen (jedoch nicht in Sachsen) ein Risiko für den Täter-Status darstellt. Interessant ist zudem, dass unter Hinzunahme des Faktors des sozioökonomischen Status` die Chance, Täter zu werden, für Migranten in Hessen noch einmal ansteigt - in Sachsen hingegen nicht. Dies könnte auf die unterschiedlichen Migrantengruppen zurückzuführen sein, die wir vorrangig in den Bundesländern finden.

Ausgangspunkt der Analyse war zudem jeweils nur ein allgemein formuliertes Item zur Mobbing-Opfer- und Mobbing-Täter-Erfahrung, was angesichts des grossen Spektrums, in dem sich Mobbing-Erfahrungen zutragen können, nicht ausreichend erscheint. So wäre es z.B. interessant zu erfahren, welche Gründe Mobbing-Opfer und -Täter angeben und ob dabei Merkmale anderer Nationalitäten, Ethnien oder Religionen eine Rolle spielen. Im sächsischen Datensatz wurden einige dieser Variablen zusätzlich erfasst. Allerdings waren aufgrund des relativ geringen Migrantenanteils in dieser Substichprobe keine fortführenden Analysen dazu möglich. Deskriptiv zeigte sich hier lediglich, dass der Anteil der Mobbing-Erfahrungen aufgrund der Hautfarbe, Herkunft oder Religion eher selten vorkommt. Lediglich $2.1 \%$ bzw. 2.5\% gaben an, wegen ihrer Hautfarbe /Nationalität oder ihrer Religion gemobbt wurden zu sein. Mobbing-Täter geben in $4.6 \%$ der Fälle an, wegen Hauttfarbe/Nationalität zu mobben; bei $2.7 \%$ ist die Religion des Opfers der Anlass. In zukünftigen Studien sollte dieser Ansatz des kulturell-diskriminierenden Mobbings unter Einbezug einer grösseren, repräsentativen Stichprobe genauer verfolgt werden, da hierdurch etwaige Ansatzstellen für Präventionsmassnahmen gewonnen werden könnten.

Es bleibt auch festzuhalten, dass die «klassischen» Variablen auf der individuellen Ebene, die die Mobbing-Täterschaft charakterisieren, nämlich männliches Geschlecht und höheres Alter, die bei weitem stärkste und beständigste Aussagekraft haben, was sich mit den Ergebnissen anderer Studien deckt. Zu den Variablen auf Klassen- und Schulebene, die hier nicht untersucht werden konn- 
ten, gehören auch das Klassen- und Schulklima. In anderen Studien erwiesen sich diese Faktoren für das Mobbing als relevant (Melzer \& Bilz, 2006). Besonders im Zusammenhang mit dem vielfach zitierten Präventionansatz von Olweus (2002) in dem auf diese Ebenen ein eigener Fokus gelegt wird, scheinen diese Variablen jedoch für Mobbing-Erfahrungen relevant zu sein. Daher sollten sie Gegenstand künftiger Mobbing-Forschung sein.

Abschliessend muss bemerkt werden, dass sich die Angaben zum Mobbing in der vorliegenden Studie vermutlich nicht zwangsläufig auf die Schule als ausschliesslichen Ort beziehen. Schüler können die berichteten Mobbing-Erfahrungen auch in anderen schulnahen Kontexten wie dem Schulweg oder auf Plätzen in der Umgebung der Schule, an denen sich die Schüler vor und nach der Schule häufig aufhalten, gesammelt haben. Dies legt, wie auch der Blick auf weitere Konstituentien in der Familie und der alltäglichen Lebenswelt der Heranwachsenden, einen Setting-Ansatz von Prävention nahe (Bals, Hanses \& Melzer, 2008). Bezüglich der Tatsache, dass ein hoher Migrantenanteil Mobbing nicht fördert, wäre es interessant, mittels weiterführender, systematischer Untersuchungen herauszufinden, welchen Wirkmechanismen Mobbing in interethnischen Kontexten unterliegt um daraus mögliche Präventionsmassnahmen abzuleiten.

\section{Anmerkungen}

1 Es lagen nur für Sachsen Daten zur Schulform vor.

2 Da die Referenzkategorie «keine Migranten in der Klasse» äusserst schwach besetzt ist, sollte man auch bei zurückhaltender Interpretation sagen können, dass der Migrantenanteil in der Klasse zumindest keinesfalls eine Erhöhung der Chance als Mobbing-Täter in Erscheinung zu treten, bedeutet.

\section{Bibliographie}

Allport, G. W. (1971). Die Natur des Vorurteils. Köln: Kiepenheuer \& Witsch.

Bals, T., Hanses, A. \& Melzzer, W. (Hrsg). (2008). Gesundheitsförderung in pädagogischen Settings - Ein Überblick über Präventionsansätze in zielgruppenorientierten Lebenswelten. Weinheim. Juventa.

Bos, W., Lankes, E.-M., Prenzel, M., Schwipper, K., Walter, G. \& Valtin, R. (Hrsg.). (2003). Erste Ergebnisse aus IGLU - Schülerleistungen am Ende der vierten Jahrgangsstufe im internationalen Vergleich. Münster: Waxmann.

Bortz, J. (2005). Statistik für Human- und Sozialwissenschaftler. Heidelberg: Springer Medizin Verlag.

Boyce, W., Torsheim, T., Currie, C. \& Zambon, A.(2006). The family affluence scale as a measure of National Wealth: Validation of an adolescent self-report measure. Social Indicators Research 78, 473-487.

Erhart, M., Schenk, L. \& Ravens-Sieberer, U. (2008). Migration und gesundheitliche Ungleichheit im Kindes- und Jugendalter. In M. Richter, K. Hurrelmann, A. Klocke \& W. Melzer (Hrsg.), Gesundheit, Ungleichheit und jugendliche Lebenswelten: Ergebnisse der zweiten internationalen Vergleichsstudie im Auftrag der Weltgesundheitsorganisation WHO (S. 151-170). Weinheim: Juventa. 
Esser, H. (2001). Integration und ethnische Schichtung. Arbeitspapier Nr. 40. Mannheimer Zentrum für Europäische Sozialforschung.

Fritzsche, S. \& Wiezorek, C. (2006). Interethnische Kontakte und Ausländerstereotype von Jugendlichen. Diskurs Kindheits- und Jugendforschung, 1, 59-74.

Fuchs, M. (1997). Ausländische Schüler und Gewalt an Schulen. In H.-G. Holtappels, W. Heitmeyer, W. Melzer \& K.-J. Tillmann (Hrsg.), Forschung über Gewalt an Schulen - Erscheinungsformen und Ursachen, Konzepte und Prävention (S. 119-136). Weinheim: Juventa.

Hradil, S. (2005). Soziale Ungleichheit in Deutschland. Wiesbaden: VS-Verlag.

Jonas, K. (1998). Die Kontakthypothese: Abbau von Vorurteilen durch Kontakt mit Fremden? In M. E. Oswald, U. Steinvorth (Hrsg.), Die offene Gesellschaft und ihre Fremden (S. 129-156). Bern: Huber.

Kriminologisches Forschungsinstitut Niedersachsen. (2005). Gewalterfahrungen von Kindern und Jugendlichen - Ergebnisse der Schülerbefragung im Jahr 2005 und Möglichkeiten erfolgversprechender Prävention. Zugriff am 19. Mai 2008 unter http://www.kfn.de/versions/kfn/assets/fb100.pdf

Melzer, W. \& Bilz, L. (2006). Familiäre Lebensverhältnisse und Sozialverhalten von Heranwachsenden: Empirische Befunde und Vorschläge für die Prävention. In T. Feltes, C. Pfeiffer \& G. Steinhilper (Hrsg.), Kriminalpolitik und ihre wissenschaftlichen Grundlagen (S. 1071-1093). Heidelberg: C. F. Müller.

Melzer, W., Bilz, L. \& Dümmler, K. (2008). Mobbing und Gewalt in der Schule im Kontext sozialer Ungleicheit. In M. Richter, K. Hurrelmann, A. Klocke, \& W. Melzer (Hrsg.), Gesundheit, Ungleichheit und jugendliche Lebenswelten: Ergebnisse der zweiten internationalen Vergleichsstudie im Auftrag der Weltgesundheitsorganisation WHO (S. 116-140). Weinheim: Juventa.

Nachtigall, C. \& Wirtz, M. (1998). Wahrscheinlichkeitsrechnung und Inferenzstatistik - Statistische Methoden für Psychologen, Teil 2. Weinheim: Juventa.

Olweus, D. (2002). Gewalt in der Schule. Was Lehrer und Eltern wissen sollten - und tun können. Bern: Huber.

Phinney, J. S., Ferguson, D. L., Tate, J. D. (1997). Intergroup attitudes among ethnic minority adolescents: A causal model. Child Development, 68 (5), 955-969.

PISA-Konsortium Deutschland (Hrsg.). (2007). PISA '06 - Die Ergebnisse der dritten internationalen Vergleichsstudie. Münster: Waxmann.

Popp, U. (2000). Gewalt in der Schule als "Türkenproblem»? Gewaltniveau, Wahrnehmung von Klassenklima und sozialer Diskriminierung bei deutschen und türkischen Schülerinnen und Schülern. Empirische Pädagogik, 14 (1), 59-91.

Schlack, R. \& Hölling, H. (2007). Gewalterfahrungen von Kindern und Jugendlichen im subjektiven Selbstbericht - Erste Ergebnisse aus dem Kinder- und Jugendgesundheitssurvey (KiGGS). Bundesgesundheitsblatt - Gesundheitsforschung - Gesundheitsschutz, 50, 819826.

Seals, D. \& Young, J. (2003). Bullying and victimisation: Prevalence and relationship to gender, grade level, ethnicity, self-esteem, and depression, Adolescence, 38 (152), 735-747.

Stein, J. A., Dukes, R. L., Warren, J. I. (2007). Adolescent male bullies, victims, and bully-victims: A comparison of psychosocial and behavioral characteristics. Journal of Pediatric Psychology, 32 (3), 273-282.

Strohmeier, D. \& Spiel, Ch. (2005). Interkulturelle Beziehungen an Wiener Schulen (IBW): Freundschaften und Feindschaften in multikulturellen Schulklassen. Institut für Wirtschaftspsychologie, Bildungspsychologie und Evaluation. Arbeitsbereichs Bildungspsychologie \& Evaluation. Universität Wien. Zugriff am 14. November 2008 unter http://www.evaluation.ac.at/download/bericht_end_interkulturelle_beziehungen.pdf 
Strohmeier, D., Atria, M. \& Spiel, C. (2005), Bullying und Viktimisierung in multikulturellen Schulklassen. Wer ist betroffen? Wie begründen Opfer ihre Erfahrungen? In A. Ittel \& M. v. Salisch (Hrsg.), Lügen, Lästern, Leiden lassen. Aggressives Verhalten von Kindern und Jugendlichen (S. 204-219) Stuttgart: Kohlhammer.

Strohmeier, D., Nestler, D. \& Spiel, Ch. (2005). Freundschaftsmuster, Freundschaftsqualität und aggressives Verhalten von Immigrantenkindern in der Grundschule. Diskurs Kindheits- und Jugendforschung, 1, 21-37.

Tropp, L. R. \& Prenovost, M. A. (2008). The role of intergroup contact in predicting children's interethnic attitudes. In S. R. Levy \& M. Killen (Hrsg.), Intergroup attitudes and relations in childhood and adulthood (pp. 236-248). Oxford: University Press.

Walter, O. \& Taskinen, P. (2007). Kompetenzen und bildungsrelevante Einstellungen von Jugendlichen mit Migrationshintergrund in Deutschland: ein Vergleich mit ausgewählten OECD-Staaten. In M. Prenzel, C. Artelt, J. Baumert, W. Blum, M. Hammann, E. Klieme \& R. Pekrun (PISA-Konsortium Deutschland, Hrsg.), PISA 2006 - Ergebnisse der dritten internationalen Vergleichsstudie (S. 337-366). Münster: Waxmann.

Schlagworte: Mobbing, Migrationshintergrund, Schule, interethnische Zusammensetzung

\section{La violence à l'école - Les relations entre l'hétérogénéité de la classe et les expériences de harcèlement moral}

\section{Résumé}

Cette étude fournit une description des expériences de harcèlement moral dans les écoles des Länder de Hesse et de Saxe à partir des données de la recherche de l'HBSC réalisée en 2006. Elle examine aussi dans quelle mesure ces expériences sont l'effet de l'origine d'immigration des étudiants et d'autres variables individuelles ainsi que des variables indiquant certaines caractéristiques des classes et des établissements scolaires.

Un tiers des élèves rapporte avoir été victime d'expériences de harcèlement moral et un quart révèle avoir été acteur de harcèlement. La position d'acteur dépend du genre et de l'âge des élèves, ainsi que du degré d'hétérogénéité culturelle (pourcentage d'immigrés) dans la classe. L'origine d'immigration semble contribuer au phénomène mais uniquement dans le Land de Hesse. Quant à la position de victime de harcèlement, elle est plus fréquente chez les étudiants de milieu socioéconomique défavorisé. Le degré d'hétérogénéité de la classe a un effet positif sur les expériences observées dans le Land de Hesse mais des effets ambigus dans le Land de Saxe.

Mots clés: harcèlement moral, école, origine d'immigration, hétérogénéité interculturelle 


\section{La violenza tra gli allievi - La relazione tra esperienze di eterogeneità e di mobbing.}

\section{Riassunto}

Questo studio fornisce una descrizione delle esperienze di mobbing negli stati federali germanici del Hessen e del Sachsen, facendo riferimento ai dati dell'inchiesta HBSC del 2006. L'attenzione verte in particolare sull'influenza rivestita, oltre che dall'origine d'immigrazione degli allievi, da altre variabili rispettivamente a livello individuale, a livello della classe e della scuola.

Un terzo degli allievi affermano di essere stati vittime di esperienze di mobbing, mentre un quarto si è detto attore di mobbing. Il sesso, l'età, così come l'eterogeneità e la parte di migranti presenti nella classe sono determinanti per quel che riguarda il ruolo di attore.

L'origine migratoria sembra contribuire al fenomeno ma unicamente nel Land di Hessen. Mentre per quel che riguarda la posizione di vittima, essa è più frequente presso gli studenti che provengono da un ambiente sfavorito. Il grado di eterogeneità della classe ha avuto un effetto positivo sulle esperienze osservate in Hessen, mentre gli effetti per il Sachsen presentano una certa ambiguità.

Parole chiave: mobbing, scuola, origine migratoria, allievi, eterogeneità interculturale.

\section{Violence in schools: The relationship between classroom heterogeneity and mobbing-incidents}

\section{Summary}

This study reviews characteristics of mobbing incidents in the federal states of Hesse and Saxony, using data collected by the HBSC-Study in 2006. It investigates to what extend, beyond the students' immigration background, other individual variables, as well as classroom and school variables may have an impact on mobbing incidents.

Approximately one third of the students indicate having sometimes been victim of mobbing, and approximately one out of four students reports having already committed this kind of offence. Results reveal that gender, age, as well as the immigrant ratio of the student's class are relevant factors to mobbing commitment. The immigration background also has an impact but only in Hesse. Students with low socioeconomic status have a higher risk of being victims of mobbing. Results reveal that classroom ethnical diversity has a positive effect in Hesse but ambiguous effects in Saxony.

Key words: mobbing, school, immigration background, ethnical diversity 